\title{
Isothermal DC and Microwave Characterizations of Power RF Silicon LDMOSFETs
}

\author{
Siraj Akhtar, Patrick Roblin, Member, IEEE, Sunyoung Lee, and Jeffrey Strahler
}

\begin{abstract}
Presented in this paper are two new approaches for the acquisition of both iso-thermal dc current-voltage $(I-V$ ) characteristics and microwave $S$-parameters of power RF LDMOSFETs. In the first approach, three-dimensional (3-D) tensor product $B$-spline representation is used to extract iso-thermal dc $I-V$ characteristics from dc $I-V$ characteristics measured at various substrate temperatures. The average device surface temperature is measured using an infrared sensor. A single effective thermal resistance is found to map the entire electrothermal profile of the device justifying the iso-thermal dc $I-V$ definition used. In the second approach, iso-thermal $I-V$ and microwave data are directly measured with an efficient procedure that keeps the average device surface temperature constant. Excellent agreement is obtained between the numerical extraction and the direct measurement approach. Finally, the comparison of the transconductance extracted from the iso-thermal dc $I-V$ and microwave data confirms the presence of a small low-frequency dispersion in LDMOSFET not due to self-heating.
\end{abstract}

Index Terms-electrothermal modeling, isothermal IV characteristic, LDMOSFET, low-frequency dispersion, pulsed IV, selfheating.

\section{INTRODUCTION}

$\mathbf{P}$ ACKAGED silicon power LDMOSFET transistors are finding increasing use for RF power amplifier design in medium- to high-power applications. Due to the high power which can be dissipated in these devices, of particular concern is the measurement and modeling of their electrothermal behavior [1]. For such modeling, isothermal microwave data are required to extract the FET model [2] and isothermal current-voltage $(I-V)$ data to determine the quiescent operating point of the device in the presence of self-heating and self-biasing. Under dc operation the dc $\left(I_{\mathrm{D}, \mathrm{DC}}\right)$ and isothermal $\left(I_{\mathrm{D}, \text { iso }}\right) I-V$ drain currents are related by

$$
\begin{aligned}
I_{\mathrm{D}, \mathrm{DC}}\left(V_{\mathrm{GS}}, V_{\mathrm{DS}}, T_{\mathrm{sub}}\right) & =I_{\mathrm{D}, \text { iso }}\left(V_{\mathrm{GS}}, V_{\mathrm{DS}}, T_{\mathrm{dev}}\right) \\
\text { with } T_{\mathrm{dev}} & =T_{\text {sub }}+R_{\mathrm{th}, \mathrm{eff}}\left(T_{\mathrm{sub}}\right) \times P_{\mathrm{avg}}
\end{aligned}
$$

where

$$
\begin{array}{ll}
T_{\text {sub }} & \text { substrate temperature; } \\
T_{\mathrm{dev}} & \text { average device temperature; } \\
R_{\mathrm{th}, \mathrm{eff}} & \text { effective thermal resistance; } \\
P_{\mathrm{avg}} & \text { average power dissipated by the FET. }
\end{array}
$$

Manuscript received January 30, 2001; revised May 7, 2001. The review of this paper was arranged by Editor K. Shenai.

S. Akhtar, P. Roblin, and S. Lee are with the Department of Electrical Engineering, Ohio State University, Columbus, OH 43210 USA (e-mail: roblin.1@osu.edu).

J. Strahler is with Lucent Technologies, Columbus, OH 43213 USA.

Publisher Item Identifier S 0018-9383(01)10116-4.

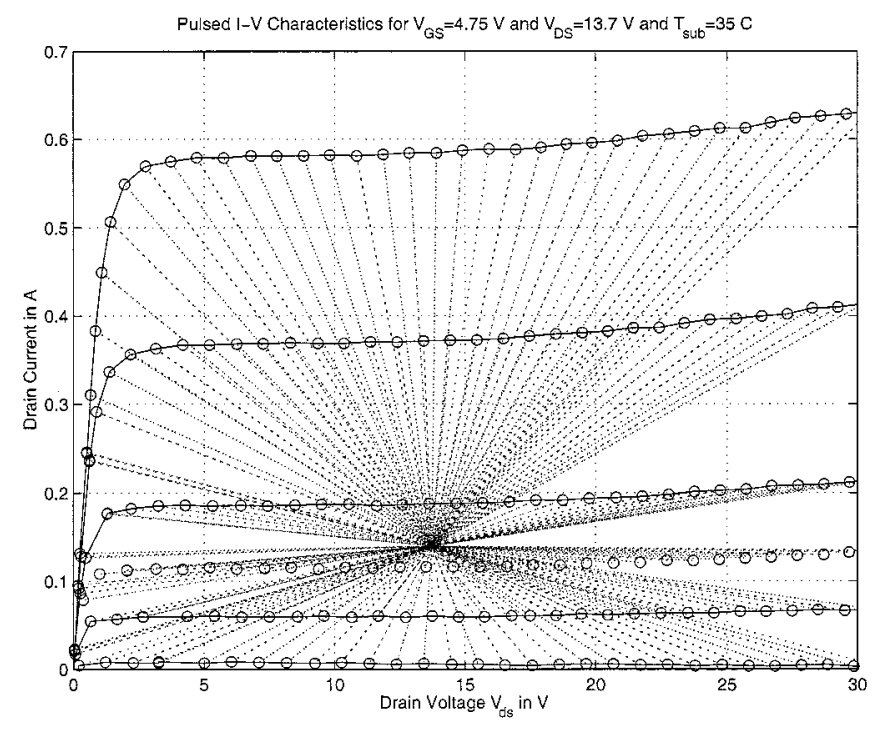

Fig. 1. Effective trajectory for pulsed $I-V$ measured in our laboratory showing the starting dc bias point. Different pulsed $I-V$ curves result if a different dc bias point is used.

In the presence of strong self heating, such as in class $B$ and $A B$ amplifiers, the isothermal $I-V$ characteristics which are a function of the device temperature must be used instead of the dc $I-V$ characteristics which are a function of the substrate temperature only.

Note that in general pulsed $I-V$ characteristics $I_{\mathrm{D} \text {,puls, }}$ are not the same as the isothermal $I-V$ characteristics $I_{\mathrm{D} \text {,iso. }}$ Indeed pulsed $I-V$ characteristics have a double bias dependence $I_{\mathrm{D}, \mathrm{puls}}\left(v_{\mathrm{GS}}, v_{\mathrm{DS}}, V_{\mathrm{GS}, \mathrm{DC}}, V_{\mathrm{DS}, \mathrm{DC}}, T_{\mathrm{dev}, \mathrm{DC}}\right)$, as they are a function of both the instantaneous voltages $v_{\mathrm{GS}}$ and $v_{\mathrm{DS}}$ and the dc bias $V_{\mathrm{GS}}$ and $V_{\mathrm{DS}}$. To illustrate this feature, we show in Fig. 1 the measurement trajectory for the pulsed $I-V$ characteristics which clearly identifies the dc bias point. Typically different pulsed $I-V$ characteristics result if a different dc bias point is used. Pulsed $I-V$ characteristics cannot therefore be equivalent to the uniquely defined isothermal $I-V$ characteristics.

To investigate the difference between pulsed $I-V$ and isothermal $I-V$ curves, consider the case of SOI-MOSFETs which are known to exhibit large low-frequency dispersions (large low-frequency dependence of its small-signal parameters) resulting in notably different $g_{\mathrm{m}}$ and $g_{\mathrm{d}}$ extracted at dc and RF-microwave. The behavior of SOI-MOSFETs can be well modeled by the electrothermal model shown in Fig. 2 [4]. This model is based upon a physical topology combining a FET model and a parasitic bipolar-transistor [3], [4] with a floating base driven by the impact ionization current. The 


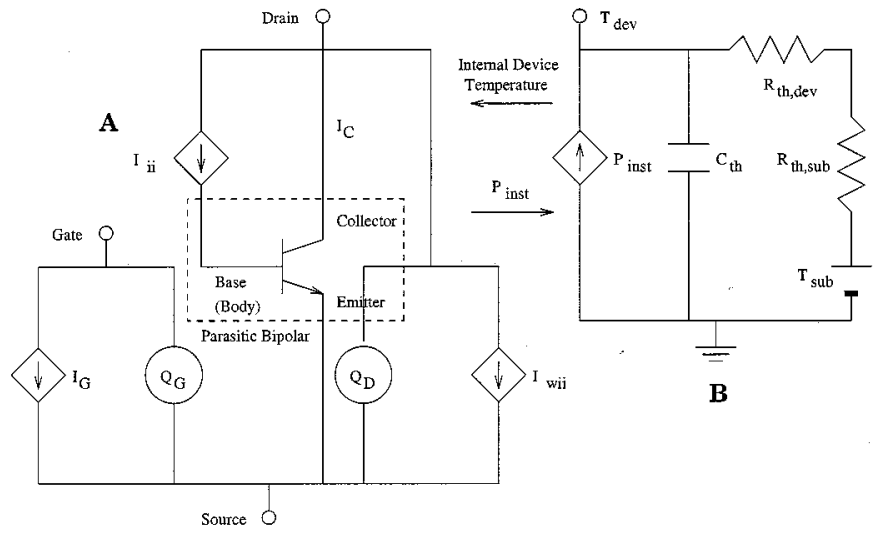

Fig. 2. (a) Self-biasing model topology to fit both dc and RF and (b) electrical network representing the thermal network model.

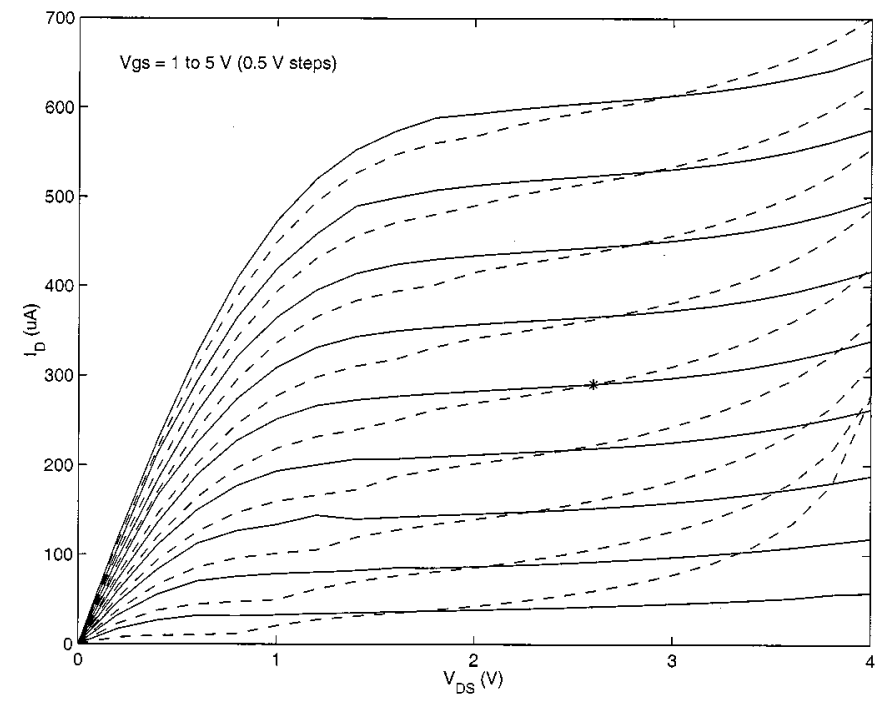

Fig. 3. Comparison of isothermal $I_{\mathrm{D}, \text { iso }}$ (dashed lines) and pulsed $I_{\mathrm{D}, \text { puls }}$ (solid lines) $I-V$ characteristics. Both curves intercept at the dc bias-point used by the pulsed $I-V$ which is indicated by an asterisk. The isothermal $I-V$ curves feature a kink when the parasitic bipolar transistor turns on.

total FET-drain current measured is therefore the sum of the bipolar-transistor collector-current $I_{\mathrm{C}}$, the impact ionization current $I_{\mathrm{ii}}$, and the normal FET drain-current without-impact-ionization $I_{\text {wii }}$. The parasitic bipolar-transistor which introduces part of the low-frequency dispersions in SOI-MOSFETs, performs with its long time response the seam-less integration between 1) the isothermal dc $I-V$ characteristics

$$
\begin{array}{r}
I_{\mathrm{D}, \text { iso }}\left(V_{\mathrm{GS}}, V_{\mathrm{DS}}, T_{\mathrm{dev}}\right)=I_{\mathrm{wii}}\left(V_{\mathrm{GS}}, V_{\mathrm{DS}}\right)+I_{\mathrm{ii}}\left(V_{\mathrm{GS}}, V_{\mathrm{DS}}\right) \\
+I_{\mathrm{C}}\left(V_{\mathrm{DS}}, I_{\mathrm{ii}}\left(V_{\mathrm{GS}}, V_{\mathrm{DS}}\right)\right)
\end{array}
$$

which sets the transistor's dc biasing and 2) the pulsed $I-V$ characteristics

$$
\begin{aligned}
& I_{\mathrm{D}, \mathrm{puls}}\left(v_{\mathrm{GS}}, v_{\mathrm{DS}}, V_{\mathrm{GS}, \mathrm{DC}}, V_{\mathrm{DS}, \mathrm{DC}}, T_{\mathrm{dev}, \mathrm{DC}}\right) \\
& =I_{\mathrm{wii}}\left(v_{\mathrm{GS}}, v_{\mathrm{DS}}\right)+I_{\mathrm{ii}}\left(v_{\mathrm{GS}}, v_{\mathrm{DS}}\right) \\
& \quad+I_{\mathrm{C}}\left(v_{\mathrm{DS}}, I_{\mathrm{ii}}\left(V_{\mathrm{GS}, \mathrm{DC}}, V_{\mathrm{DS}, \mathrm{DC}}\right)\right)
\end{aligned}
$$

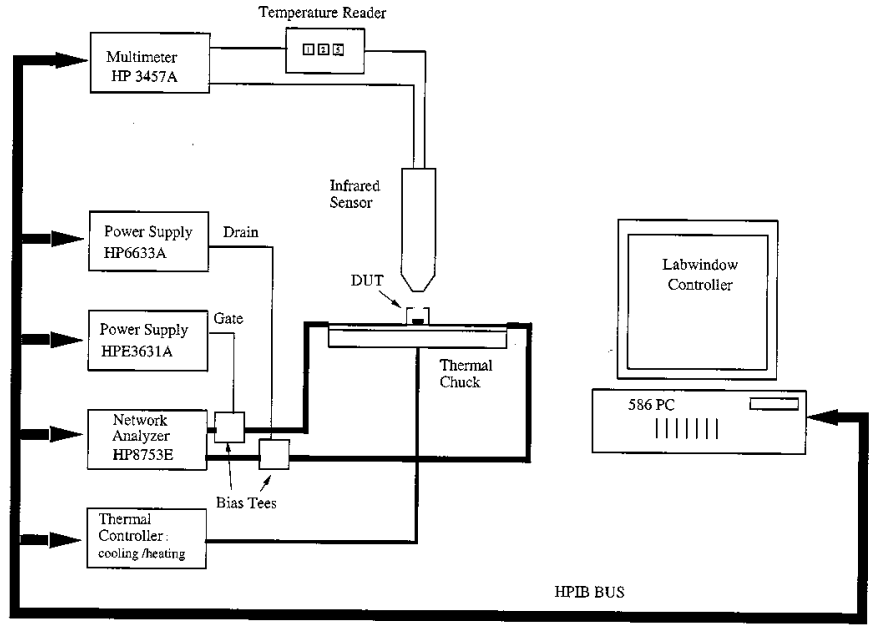

Fig. 4. Testbed used for measuring the isothermal $I-V$ characteristics and microwave $S$-parameters.

which are relevant to the power transistor's response at microwave frequencies. Note that these $I-V$ curves intersect at the dc bias-point $\left(V_{\mathrm{GS}}, V_{\mathrm{DS}}\right)$

$$
\begin{aligned}
I_{\mathrm{D}, \text { puls }}\left(v_{\mathrm{GS}}=V_{\mathrm{GS}}, v_{\mathrm{DS}}\right. & \left.=V_{\mathrm{DS}}, V_{\mathrm{GS}}, V_{\mathrm{DS}}, T_{\mathrm{dev}}\right) \\
& =I_{\mathrm{D}, \mathrm{iso}}\left(V_{\mathrm{GS}}, V_{\mathrm{DS}}, T_{\mathrm{dev}}\right) .
\end{aligned}
$$

As is shown in Fig. 3 for a SOI-MOSFET, the pulsed $I-V$ curves, which are also isothermal, and the isothermal $I-V$ curves are usually different (apart from the bias-point) because the pulsed $I-V$ bypass not only self-heating but also other slow physical processes such as parasitic bipolar-transistor and trap effects which induce additional low-frequency dispersion.

In line with this discussion, we present below new methods for obtaining isothermal $I-V$ characteristics and isothermal microwave $S$-parameters without using pulsed measurements.

\section{DC THERMAL CHARACTERIZATIONS}

We have used for this entire work Motorola's MRF 181 RF power, $n$-channel enhancement mode, lateral diffused MOSFET [5]. The electrothermal test system shown in Fig. 4 was used to obtain a complete dc and thermal profile of the LDMOSFET. LabWindows [6] is used to control $V_{\mathrm{DS}}, V_{\mathrm{GS}}$, and the substrate temperature $T_{\text {sub }}$, while measuring the resulting non-isothermal drain current $I_{\mathrm{D}, \mathrm{DC}}$ and average device temperature $T_{\mathrm{dev}}$. A full set of $I-V-T_{\mathrm{dev}}$ curves are obtained for various $T_{\text {sub }}$ values. The top part of the packaging of the transistor has a small hole drilled through to allow for an infrared thermometer (Mikron M67S0000-030 CL-3S Infraducer 2-Wire) to focus on the device surface in order to measure $T_{\mathrm{dev}}$. The infrared thermometer is first calibrated over the measurement range with the FET unbiased $\left(T_{\mathrm{sub}}=T_{\mathrm{dev}}\right)$, by adjusting the surface emissivity setting till the thermometer correctly predicts (within $\pm 1.2 \%$ ) the temperature set by the thermal chuck controller.

Fig. 5 shows the measured $I-V$ for a $T_{\text {sub }}$ of $29^{\circ} \mathrm{C}$. The resulting device temperature is superimposed at each bias-point showing $T_{\text {dev }}$ exceeding $170{ }^{\circ} \mathrm{C}$ for high bias values. Also at high bias, the drain current is observed to decrease as a result of the self-heating in the device. 


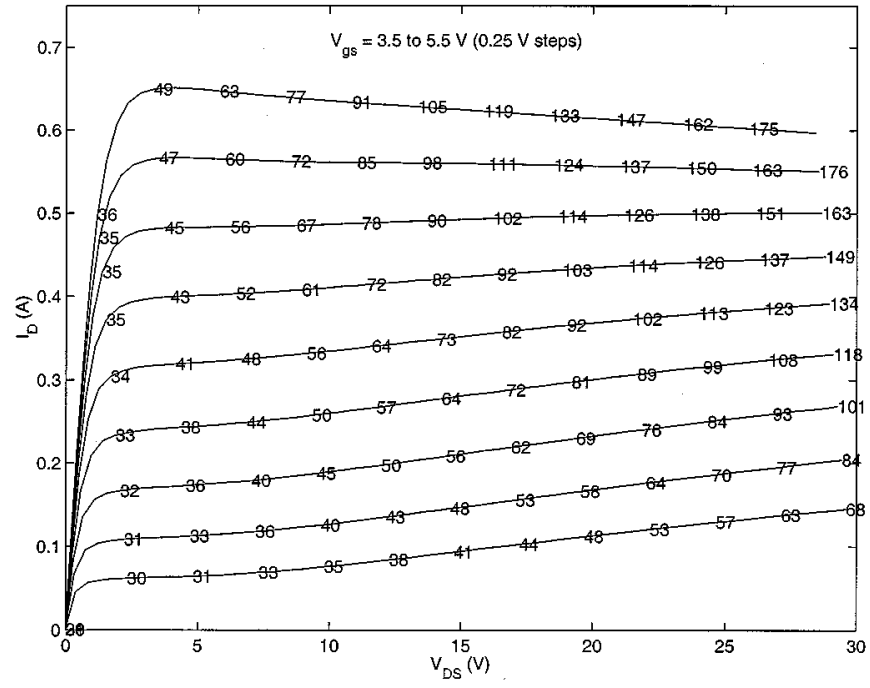

Fig. 5. Measured $I-V$, with $T_{\mathrm{dcv}, \text { avg }}$ superimposed, for a constant substrate temperature of $29^{\circ} \mathrm{C}$.

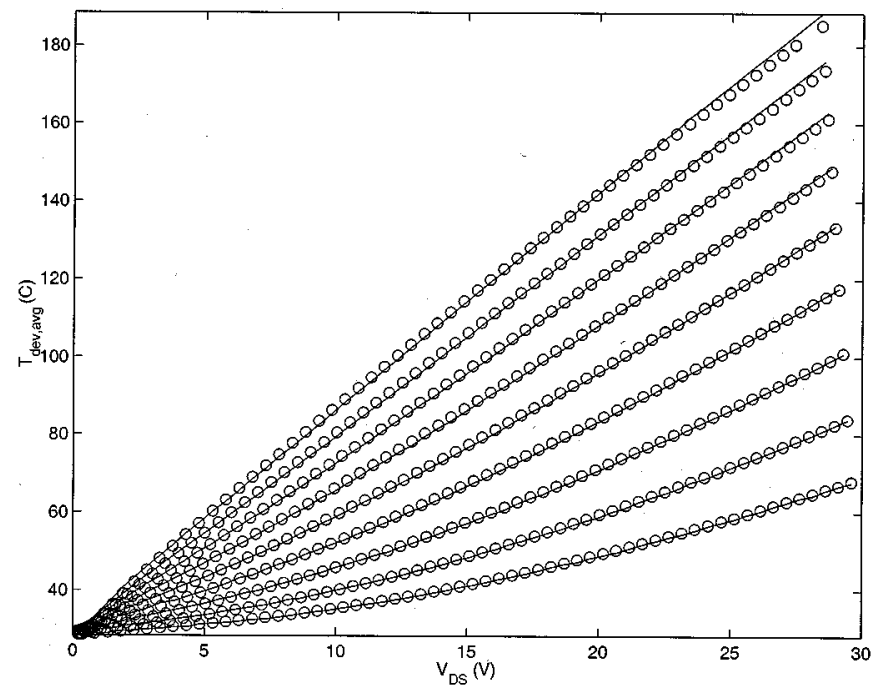

Fig. 6. Predicted average device temperature (solid lines) compared with measured values (circles) using a single $R_{\mathrm{tll}, \mathrm{cff}}$ for a given $T_{\mathrm{sub}}$ of $29^{\circ} \mathrm{C}$.

The large-signal electrothermal model for the LDMOSFET shown in Fig. 2, features a simple thermal network topology which calculates the steady state isothermal temperature of the LDMOSFET as a function of the power dissipated by the LDMOSFET.

An effective thermal resistance $R_{\mathrm{th}, \text { eff }}$ (the sum of $R_{\mathrm{th}, \mathrm{dev}}$ and $R_{\mathrm{th}, \mathrm{sub}}$ ), can be obtained by using a least squares fit on the temperature versus device power characteristics. A unique $R_{\mathrm{th}, \text { eff }}$ value is extracted for each $T_{\mathrm{sub}}$ and is found to be 9.26 ${ }^{\circ} \mathrm{C} / \mathrm{W}$ for $T_{\text {sub }}=29^{\circ} \mathrm{C}$. At $T_{\text {sub }}=89^{\circ} \mathrm{C}, R_{\text {th,eff }}$ equals 8.74 ${ }^{\circ} \mathrm{C} / \mathrm{W}$. The effective thermal resistance is observed to be monotonously decreasing with increasing substrate temperature. Using this $R_{\mathrm{th}, \mathrm{eff}}$ value, we can compute the average device temperature for any bias. The solid lines in Fig. 6 show the predicted $T_{\text {dev }}$ for a $T_{\text {sub }}$ of $29^{\circ} \mathrm{C}$. The circles give the measured $T_{\mathrm{dev}}$. Clearly, a single $R_{\mathrm{th}, \mathrm{eff}}$ (see [7] and [8]) can predict well the entire thermal map of the $1-\mathrm{mm}^{2}$ device tested despite its

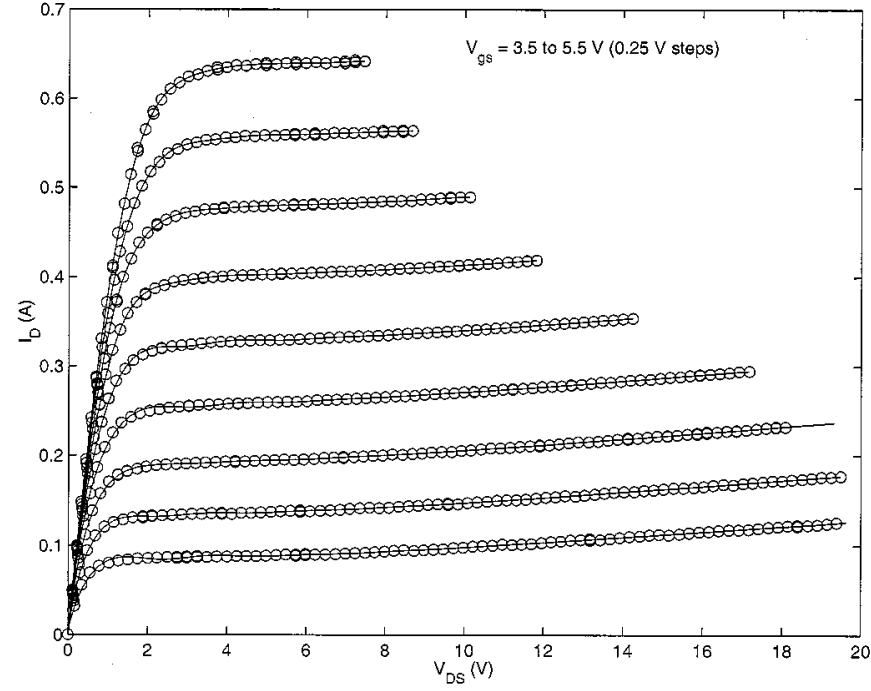

Fig. 7. Tensor Product B-Splines (TPS) extracted (solid lines) and directly measured (circles) isothermal $I-V$ for $T_{\mathrm{ccv}}=90{ }^{\circ} \mathrm{C}$ with both substrate cooling and heating.

relatively large area. This also verifies the applicability of the thermal model used for defining the isothermal $I-V$ in (1).

\section{EXTRACTION AND MEASUREMENT OF ISO-THERMAL $I-V$ CURVES}

\section{A. Numerical Extraction}

Using three-dimensional (3-D) Tensor Product B-Splines (TPS) [9], [10], a function of three variables can be represented as

$$
\begin{aligned}
& I_{\mathrm{D}, \mathrm{iso}}\left(V_{\mathrm{GS}}, V_{\mathrm{DS}}, T_{\mathrm{dev}}\right) \\
& =\sum_{i=1}^{m} \sum_{j=1}^{n} \sum_{l=1}^{p} a_{i j k} B_{i, k_{\mathrm{GS}}, t_{\mathrm{GS}}}\left(V_{\mathrm{GS}}\right) B_{j, k_{\mathrm{DS}}, t_{\mathrm{DS}}}\left(V_{\mathrm{DS}}\right) \\
& \quad \times B_{l, k_{\mathrm{dev}}, t_{\mathrm{dcv}}}\left(T_{\mathrm{dev}}\right) .
\end{aligned}
$$

Using data from the complete dc and thermal characterization presented in Section II, the TPS method can be used to extract a complete set of isothermal $I-V$ characteristics for different average device temperatures. In Fig. 7, the TPS extracted isothermal $I-V$ for a $T_{\mathrm{dev}}$ of $90^{\circ} \mathrm{C}$ is given by the solid lines. The isothermal $I-V$ clearly do not contain the self-heating induced reduction of the drain current observed in the non isothermal $I-V$ curves at large drain voltages. The TPS method is limited by the range of the data available. Lowering the substrate temperature will allow for data at higher biases to be available for a given $T_{\mathrm{dev}}$.

\section{B. Direct Measurement}

A novel approach to directly acquire isothermal $I-V$ characteristics and microwave data has been implemented in LabWindows. In this approach, the computer-controled data-acquisition system maintains a constant device temperature $T_{\mathrm{dev}}$ by acquiring $I-V$ and microwave data on constant power $\left(P_{\text {avg }}\right)$ contours. In the measurement procedure, the substrate temperature is first set to the lowest temperature (in this case, cooled 


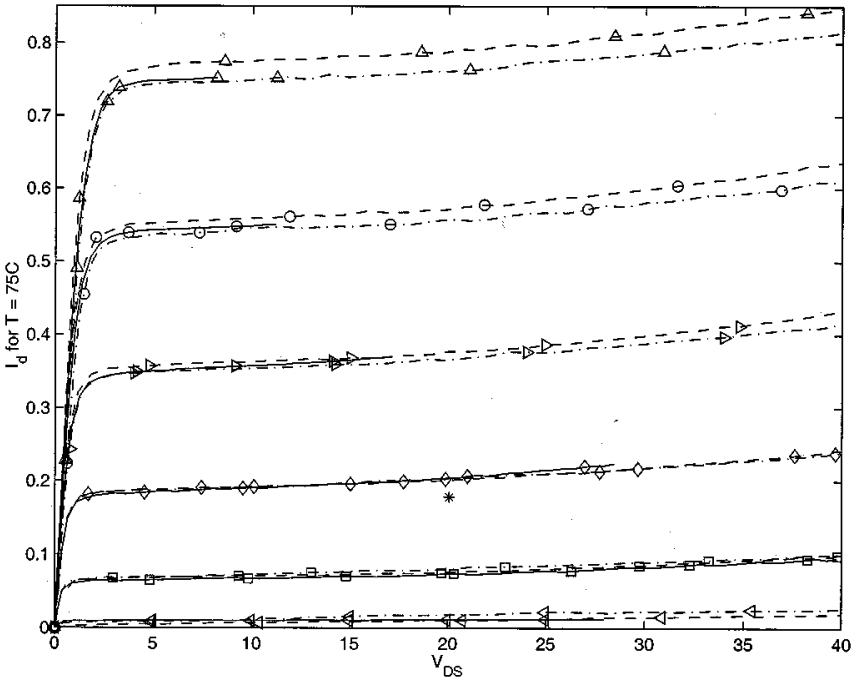

Fig. 8. Isothermal $I-V$ characteristics (solid line) and two different pulsed $I-V$ characteristics for OFF (dashed line) and ON (dashed-dotted line) dc bias-points. The OFF bias-point corresponds to $V_{\mathrm{DC}, \mathrm{ds}}=0 \mathrm{~V}$ and $I_{\mathrm{DC}, \mathrm{ds}}=0 \mathrm{~A}$. The $\mathrm{ON}$ bias point corresponds to $V_{\mathrm{DC}, \mathrm{ds}}=20 \mathrm{~V}$ and to the drain current indicated with a star. In all three $I-V$ characteristics, the device temperature is $T_{\mathrm{ccv}}=75^{\circ} \mathrm{C}$ and $V_{\mathrm{GS}}$ ranges from $4 \mathrm{~V}$ to $6.5 \mathrm{~V}$ in steps of $0.5 \mathrm{~V}$.

to $18^{\circ} \mathrm{C}$ ) and $V_{\mathrm{GS}}$ is set to its highest value. $V_{\mathrm{DS}}$ is then swept and the drain current at all data points within $\pm 2{ }^{\circ} \mathrm{C}$ of the targeted $T_{\mathrm{dev}}$ are recorded after establishing thermal equilibrium in the device (a $5 \mathrm{~s}$ pause was verified to be more than sufficient for a bias change with the same substrate temperature). $V_{\mathrm{GS}}$ is then lowered and the drain voltage sweep is repeated. Once the data has been acquired for the lowest $V_{\mathrm{GS}}$, the substrate temperature is increased each time (in this case by $4{ }^{\circ} \mathrm{C}$ with a $240 \mathrm{~s}$ delay allowed for the substrate to reach thermal equilibrium) and the $V_{\mathrm{GS}}$ and $V_{\mathrm{DS}}$ bias-sweep process is repeated. The higher the substrate temperature, the lower the drain voltages that will give the targeted device temperature $T_{\mathrm{dev}}$. This novel approach permits a rapid data acquisition since all data are acquired in a single sweep of the substrate temperature from its lowest to highest value. This procedure minimizes the impact of the thermal inertia of the substrate on the measurement time and is fully reproducible from measurement to measurement.

The circles in Fig. 7 show the directly measured $90{ }^{\circ} \mathrm{C}$ isothermal $I-V$, which is in excellent agreement with that extracted using TPS (solid lines). By cooling the substrate, the bias-range of the isothermal $I-V$ acquired is increased since for a cooler substrate, the targeted $T_{\mathrm{dev}}$ is achieved for higher bias-voltages.

Finally, the $T_{\mathrm{dev}}=75^{\circ} \mathrm{C}$ pulsed $I-V$ characteristics for two different (OFF and ON) dc bias-points are plotted in Fig. 8 together with the directly measured isothermal $I-V$ characteristics. Pulses with a duration of $1 \mu$ s and $1 \%$ duty-rate are used. The ON and OFF pulsed $I-V$ curves were shifted down by 10 $\mathrm{mA}$ to account for a difference in equipment calibration and enforce the required dc bias condition given by (4). A very good agreement is obtained between the isothermal and the $\mathrm{ON}$ pulsed $I-V$ characteristics for all gate voltages. Note however, that a substantial difference is observed between the $\mathrm{ON}$ and OFF pulse $I-V$ data, highlighting their dc bias dependence.

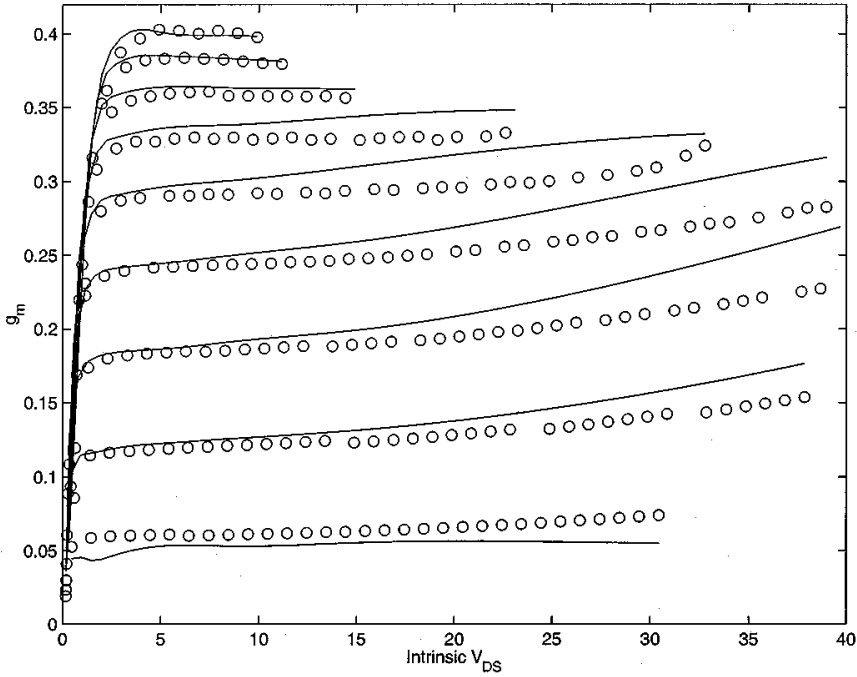

Fig. 9. Comparison of isothermal $\mathrm{DC} g_{\mathrm{m}}$ (solid lines) and $\mathrm{RF} g_{\mathrm{m}}$ (circles) for $T_{\mathrm{ccv}}$ of $90{ }^{\circ} \mathrm{C}$. The $\mathrm{DC} g_{\mathrm{m}}$ are calculated from the isothermal $I-V$ characteristics. The $\mathrm{RF} g_{\mathrm{m}}$ are extracted (see [2]) from the microwave $S$-parameter data which are acquired at the same time as the isothermal $I-V$ characteristics.

\section{EXTRACTION AND MEASUREMENT OF ISO-THERMAL $S$-PARAMETERS}

The direct isothermal measurement method introduced in Section III can also be used for simultaneously measuring the microwave $S$-parameters (see Fig. 4) while acquiring the isothermal $I-V$ characteristics. See [2] for examples of measured isothermal (same $T_{\mathrm{dev}}$ ) $S$-parameters acquired that way. The intrinsic $\mathrm{RF} g_{\mathrm{m}}$ and $\mathrm{RF} R F g_{\mathrm{d}}$ were extracted from these $S$-parameter data using the analytic procedure described in [2].

It has been verified in [4] that for SOI-MOSFETs afflicted with a strong parasitic bipolar-transistor effect (see Fig. 3), the TPS integration of intrinsic $\mathrm{RF} g_{\mathrm{m}}$ and $\mathrm{RF} g_{\mathrm{d}}$ with the dc boundary condition set by (4), yields the pulsed $I-V$ characteristics for that dc bias-point and not the isothermal dc $I-V$ characteristics. Therefore, to detect the presence of a low-frequency dispersion in LDMOSFET we compare in Fig. 9 for all biases, the transconductances extracted from the isothermal microwave $S$-parameters $\left(\mathrm{RF} g_{\mathrm{m}}\right)$ and the isothermal $I-V$ characteristics $\left(\mathrm{DC} g_{\mathrm{m}}\right)$ which are measured at the same time. A good agreement is generally observed. This indicates that in these LDMOSFETs, the body tie suppresses the low-frequency dispersion effect associated with the parasitic bipolar-transistor. However, at large voltages a noticeable difference in $\mathrm{DC} g_{\mathrm{m}}$ and $\mathrm{RF} g_{\mathrm{m}}$ is observed. This is attributed to the increase of impact-ionization current which drives the parasitic bipolar-transistor.

\section{CONCLUSION}

We have presented in this paper two new approaches for the generation of isothermal $\mathrm{dc}$ and microwave characteristics of power RF LDMOSFETs. In the first approach, TPS representation was used to extract isothermal $I-V$ data from dc $I-V$ characteristics measured at various substrate temperatures. In the second approach, isothermal $I-V$ and microwave 
characteristics are directly measured with an efficient (constant power contour) experimental procedure that keeps the average device/gate temperature constant. An excellent agreement was demonstrated between the numerical extraction and the direct measurement approach for the $I-V$ characteristics. Finally, the correlation of the isothermal dc and microwave data acquired simultaneously with the direct measurement-technique, was analyzed.

\section{ACKNOWLEDGMENT}

The authors wish to thank the reviewers for many useful suggestions. They are also grateful to Lucent Technologies for the support of this work.

\section{REFERENCES}

[1] P. Khandelwal, M. Trivedi, K. Shenai, and S. K. Leong, "Thermal and package performance limitations in LDMOSFETs for RFIC applications," IEEE Trans. Microwave Theory Tech., vol. 47, pp. 575-585, May 1999.

[2] P. Roblin, S. Akhtar, and J. Strahler, "New nonquasi-static theory for extracting small-signal parameters applied to LDMOSFETs," IEEE Microwave Guided Wave Lett., vol. 10, pp. 322-324, Aug. 2000.

[3] D. Suh and J. G. Fossum, "A physical charge-based model for nonfully depleted SOI MOSFETs and its use in assessing floating-body effects in SOI CMOS circuits," IEEE Trans. Electron Devices, vol. 42, pp. 728-737, Apr. 1995.

[4] S. Akhtar and P. Roblin, "B-spline based, large-signal DC and microwave-model for SOI-MOSFETs," in Analog Integrated Circuits and Signal Processing. Norwell, MA: Kluwer, 2000, Special issue on RF/Microwave SOI-CMOS IC, pp. 115-131.

[5] "The RF MOSFET line: RF power field effect transistor: N-channel enhancement-mode lateral MOSFET," Motorola Semiconductor Technical Data.

[6] "LabWindows/CVI: Getting started with LabWindows/CVI," National Instruments, 1996.

[7] B. M. Tenbroeak, M. S. L. Lee, W. Redman-White, J. T. Bunyan, and M. J. Uren, "Self-heating effects in SOI MOSFETs and their measurement by small-signal conductance techniques," IEEE Trans. Electron Devices, vol. 43, pp. 2240-2248, Dec. 1996.

[8] D. Heo, E. Chen, E. Gebara, S. Yoo, J. Laskar, and T. Anderson, "Temperature dependent MOSFET RF large-signal model incorporation self heating effects," in Proc. IEEE MTT-S Int. Microwave Symp. Dig., 1999, pp. 415-418.

[9] G. Bischoff and J. P. Krusius, "Technology independent device modeling for simulation of integrated circuits for FET technologies," IEEE Trans. Computer-Aided Design, vol. CAD-4, pp. 99-110, Jan. 1985.

[10] C. de Boor, A Practical Guide to Splines. New York: Springer-Verlag, 1978.

[11] J.-P. Tayssier, P. Bouysse, Z. Ouarch, D. Barataud, T. Peyretaillade, and R. Quere, "40-GHz/150-ns versatile pulsed measurement system for microwave transistor isothermal characterization," IEEE Trans. Microwave Theory Tech., vol. 46, pp. 2043-2052, Dec. 1998.

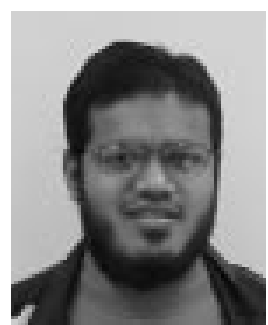

Siraj Akhtar received the B.S.E.E. degree (summa cum laude) from the University of Mississippi, University, MS, in 1993, and the M.S.E.E. and Ph.D. degrees from Ohio State University, Columbus, in 1996 and 2000, respectively. While at the University of Mississippi, he was a recipient of the Taylor Medal - the highest academic achievement award conferred by the university.

$\mathrm{He}$ was an NEC/Litton Fellow at Ohio State University for one year, after which time he became a Teaching and Research Associate. In his teaching duties, he has been involved in the laboratory sections of three microwave classes - passive circuits, active circuits, and system design. His research areas of interests include electrothermal RF and power device modeling, power amplifier and RF circuits, and SOI devices and circuits.

Dr. Akhtar is a Member of Eta Kappa Nu, Tau Beta Pi, and Phi Kappa Phi.

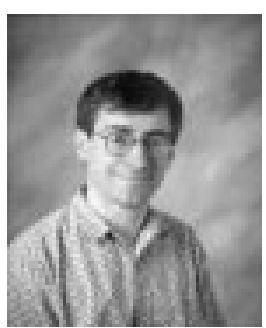

Patrick Roblin (M'85) was born in Paris, France, in September 1958. He received the Maitrise de Physics degree from the Louis Pasteur University, Strasbourg, France, in 1980, and the M.S. and D.Sc. degrees in electrical engineering from Washington University, St. Louis, MO, in 1982 and 1984, respectively.

In August 1984, he joined the Department of Electrical Engineering at Ohio State University (OSU), Columbus, as an Assistant Professor and became an Associate Professor in June 1990. He has spent the 1993-1994 academic year on professional leave at the Microelectronics and Technology Center at Allied Signal Aerospace. His research interest includes the physics of semiconductor heterostructures devices, the measurement and modeling of both high-speed and high-power semiconductor devices (RTD, MODFET, SOI-MOSFET, LDMOS) for RF/microwave circuit simulation, and the design and measurement of RF power-amplifiers and active antennas. He has also developed at OSU an educational RF/microwave laboratory and curriculum for training both undergraduate and graduate students.

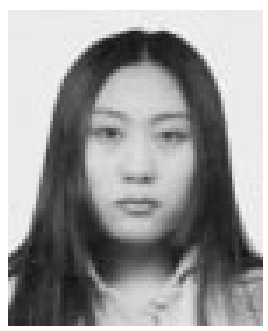

Sunyoung Lee was born in Kyoungju, Korea, in 1974. She received the B.S. degree from Korea University, Seoul, Korea in 1997. She is currently pursuing the M.S. degree at Ohio State University, Columbus. As part of her Master thesis, she has developed a pulsed $I-V$ measurement system for power FETs and made pulsed $I-V$ measurements of LDMOSFETs. She is also working on the electrothermal modeling of LDMOSFETs for power amplifier design.

Jeffrey Strahler received the B.S.E.E. degree from the University of Cincinnati, Cincinnati, OH, in 1989 and the M.S.E.E. degree in electrical engineering from The Ohio State University, Columbus, in 1991. He completed his M.S. thesis in the area of computational electromagnetics at the ElectroScience Laboratory in the Electrical Engineering Department.

In 1991 he joined AT\&T Bell Laboratories, now Lucent Technologies, Columbus, as Member of Technical Staff (MTS). Since 1999, he has been a Distinguished MTS. As part of his duties, he has been a team leader for the design and development of wireless base station amplifiers for AMPS, TDMA, and CDMA systems. 